\title{
Stochastic simulation of assembly line for optimal sequence using Petri Nets $(\mathbf{P N})$
}

\author{
${ }^{1}$ Subhash Chandra, ${ }^{2}$ Muhammad Al Salamah, ${ }^{3}$ Vakkar Ali \\ ${ }^{1,2.3}$ Department of Mechanical and Industrial Engineering, Majmaah University, K.S.A. post code 11952
}

\begin{abstract}
The assembly of any product or machine is highly complex. The order of sequence affects the make span time of assembly and thus the throughput. Which part should come in what order and what tasks will be performed together, how many work stations are required etc. are very crucial in obtaining the optimal line. The number of work stations, what task constitute the work station, which precede and what is in succession will affect the time to accomplish the task and also the utilization of resources i.e. manpower and machines both. There may be many solutions available to assembly line but it has to be validated. It is therefore argued to simulate the proposed solutions. The solution based on average data will not represent the actual plant performance. It is inevitable to have a promising technique to arrive at pragmatic result and brings down the assumptions to minimal. The stochastic nature of setup times, no operation times will affect the performance of the plant. The stochastic task timings are being used to represent real conditions and compare the performance of different alternative sequences of assembly. The assembly of finished product is the outcome of work break down (WBD), their sequence of assembly based on multiple precedence relationships. It is a highly challenging job for engineers to find the optimal sequence so that the utilization of machines and throughput are maintained high. The possible sequences of assemblies are tested and the sequence that yields maximum output must be selected. Hence one has to find the solution based on dynamic conditions. This paper is an attempt to first get solution using optimization techniques using linear programming problem (LPP) or heuristics. Then simulate those sets on Petri Nets. The Petri Net is an interactive, simple and yet very powerful technique to represent the problem graphically and coding the same using general purpose programming language for validating the same for intended result. Real time patterns using best fit, statistical interferences, probability density functions ( $p d f)$, discrete distributed systems(DDS), or stochastic modeling can be used to simulate problem to measure performance of assembly line.
\end{abstract}

Keywords: probability density function (pdf), discrete distributed systems (DDS), stochastic task times, Petri Net $(P N)$, simulation, linear programming problem (LPP).

\section{Introduction}

It is always not possible to make prototype of real system and evaluate the real time performance. A Petri Net is one of the several mathematical representations of discrete distributed systems (DDS). Petri Net (PN) has gained comprehensive role in the area of simulation and modeling for its simplicity for performance evaluation. The paper attempts to show the capabilities of Petri Net for the assembly of a product as case study for stochastic simulation.

The first work emanated as Carl Adam Petri's doctoral dissertation in 1962. A.W. Holt and others of the Information System and theory Project of Applied Research, Inc. in the United States thereafter. Their work illustrated how Petri nets could be used to model and analyze systems of many concurrent components. (Holzmann, 1991).

Starting in the late 70's, Petri nets assumed greater significance, especially in Europe. Annual conferences on Application and Theory of Petri Nets have been held since 1979 and the proceeding published in the series of lecture notes of computer science by Springer-Verlag. Professor T. Murata gave an excellent tutorial paper in 1989, which comprehensively presented properties, analysis, and application of Petri nets and a list of references of significance. The books and most of the papers were primarily targeted at computer scientists and graduate students [KP Vellanias, 2005].

There are stochastic and deterministic simulation techniques widely applicable to manufacturing, workflow management and telecommunications to aid in planning for system details (Vrat P., 1976). The manufacturing facilities are complex in terms of scheduling, sequencing, tooling requirements, uncertain failures, buffer stocks and machines waiting for materials. The number of sequences can be simulated by changing the input places and out put places matrix data used in Petri Net. This maneuverability is very effective to explore various options under stochastic task timings. In such situations Petri net turn out to be very promising and quite illustrative to represent such complex system graphically (Averill M. Law, 2000). 


\section{Basics of Assembly Line Sequencing and Balancing:}

The purpose of assembly line sequencing and balancing is to arrange the tasks and group the tasks economically along a production assembly line while observing the restrictions imposed by the nature of assembly line production in general and individual circumstances in particular in such a manner as to achieve prescribed objectives. The objective may be minimization of the work force (number of stations) or the maximization of output rate (i.e. minimization of cycle time). By balancing, an attempt is made to give each worker on the line an almost equal amount of work (in time units) so that production flows smoothly through the work stations. This has been defined in different ways in the literature by relaxing some of the assumptions of simple balancing. When assumption 8 is relaxed, a multi/mixed model case is implied (Bhattacharjee, 2007).

Definitions

Work station: A location on the assembly line where a given amount of work is performed. For any assembly line, $1<\mathrm{k}<\mathrm{K}$, where $\mathrm{K}=$ maximum number of work stations in the line.

Work element: A rational division of total work content in an assembly process. An element is represented by i, where ' $\mathrm{i}$ ' is the element number with the range $1<\mathrm{i}<\mathrm{N}$, where $\mathrm{N}$ is the total number of work elements.

Cycle time (CT): The amount of time required for a unit of product to be assembled by an operator at a work station.

Efficiency: The ratio of total station time to the product of cycle time and the number of stations. Smoothness index: An index to indicate the relative smoothness of a given assembly line balance. It consists of the square root of the sum of the squares of the time deviation for each of the stations in the line from the maximum station time. The smaller the value of the index, the better is the balance.

Lower bound (LB): The theoretical minimum number of stations for a line balancing problem. It is obtained by dividing the sum of the elemental times by the cycle time. In the case of a fraction, the next higher integer value is taken. For an optimum solution, the number of stations will be equal to or greater than the lower bound (Raouf, 1980, Buxey, G.M.1974).

Assembly line consists of assigning work elements to work stations so that a "sensible" work content for that station is established. In the proposed generalized model, the elements will be assigned to the stations subject to the following restrictions:

(A) Technological restrictions: These define relationships which may be shown diagrammatically as a precedence graph.

(B) Zoning constraints: These are the restrictions which make it desirable that two or more specific operations should or should not be performed at the same station (Agrawal PK, 1985, Bhattacharjee T.K, 1987).

\section{Mathematical Model Of Assembly Line}

The IP set identify the assembly ordering constraints like $\mathrm{IP}=\{(\mathrm{u}, \mathrm{v}),(\mathrm{)})$, this shows that task u must precede task $\mathrm{v}$. The binary indicators can be used as decision variables. If task $\mathrm{i}$ is assigned to station $\mathrm{k}$,

$$
x_{i k}=\left\{\begin{array}{cc}
1 & \text { iftaskiassignedtostationk } \\
0 & \text { ifnotso }
\end{array}\right.
$$

Jobs are strictly ordered for sequence of operations. ZS can be set of tasks that can be performed on the same station.

$$
\therefore \sum_{k=1}^{k} X_{u k} X_{v k}=1 \quad(u, v) \in Z S
$$

$$
X_{u b+} X_{v b} \leq 1, \quad b=1, \quad 2, \quad \ldots . \quad . . k(u, v) \in Z D
$$

Where ZD is the set of task pair that cannot be performed on the same workstation.

For minimum span time the objective function should look like

Minimize $\sum_{i=1}^{N} \sum_{k=1}^{k} t_{i k} X_{i k}$

Where N: Number of tasks and K: Number of stations.

Subjected to

$$
\begin{aligned}
& \sum_{i=1}^{N} t_{i k} X_{i k} \leq C, \text { for } k=1 \ldots \text { to } k \\
& \qquad \sum_{k=1}^{k} X_{i k}=1 \quad i=1, \ldots \ldots . . . \\
& \qquad X_{v b} \leq \sum_{j=1}^{b} X_{u j} b=1, \ldots k(u, v) \in I P
\end{aligned}
$$

If $\mathrm{v}$ is assigned to station 3 than $\mathrm{u}$ can be assigned to station 1,2 , and 3 . These are called zonal constraints, $\mathrm{u}$ has to be completed before $\mathrm{v}$. There are others positive constraints that is two parts will be together which is given by set ZS. There will be one more negative constraints that is two parts can not be together. Thus $X_{\mathrm{uk}}+\mathrm{X}_{\mathrm{vk}}<=1.0$ which is taken care by set ZD. There are other algorithms or heuristics like 
COMSOAL, Rank Position Weight, simulated annealing, and genetic Algorithms (Sayed et. al. 2011, Askin R.G 1993) which have been presented by researchers time and again to find better solution of line balancing and sequencing problem.

\section{Basics Of Petri Nets}

A Petri net is a bipartite directed graph that contains places represented by circles; transitions represented by boxes or bars, and directed arcs connecting places to transitions and transitions to places. The movement of tokens represents the dynamic nature of the modeled system in a Petri net. A token, represented as a small dot, is placed in a circle to indicate that the state is active. The current location and distribution of tokens in a Petri net is called its marking. The marking of the Petri net defines the state of the system. A Petri net containing tokens is called a marked Petri net [ Michel Diaz, 2005, Peter Haas, 2010 Chandra, 2008].

\section{FORMAL DEFINITION OF PETRI NETS}

Ordinary $\mathrm{PN}$ can be formally defined as a fourtuple: $P N=(P, T, I, O)$ where: $\mathrm{P}$ is a finite non-empty set of places. $P=\{\mathrm{p} 1, \mathrm{p} 2, \ldots, \mathrm{pn}\}$, Tis a finite non-empty set of transitions. $T=\{\mathrm{t} 1, \mathrm{t} 2, \ldots, \mathrm{ts}\}, \mathrm{I}$ is an input function. $\mathrm{I}:(\mathrm{P} \times \mathrm{T}) \rightarrow\{0,1\}, \mathrm{I}(p, t)=1$

if there exists an arc from $\mathrm{P}$ to $\mathrm{T}$. If so, pis an input place for $\mathrm{t}$.

Ois an output function. O: $(\mathrm{T} \times \mathrm{P}) \rightarrow\{0,1\}, O(t, p)=1$ if there exists an arc from $t$ to $p$. If so, $p$ is an output place for $\mathrm{t}$; $\mathrm{M}: \mathrm{P} \square \square N$ is a marking that assigns a non-negative

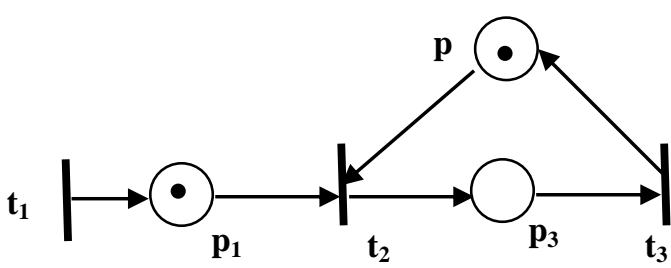

Fig.1 a simple PN example integer to each place. Such a number represents the number of tokens in the place. M represents a marked Petri net. The details for figure 1 are presented by the followings. Places: $P=\left\{p_{1}, p_{2}, p_{3}\right\}$; Transitions: $T=\left\{t_{1}, t_{2}, t_{3}\right\}$; Input places: $\mathrm{I}\left(\mathrm{t}_{1}\right)=\{\}, \mathrm{I}\left(\mathrm{t}_{2}\right)=\left\{\mathrm{p}_{1}, \mathrm{p}_{2}\right\}, \mathrm{I}\left(\mathrm{t}_{3}\right)=\left\{\mathrm{p}_{3}\right\}$; Output places: $\mathrm{O}\left(\mathrm{t}_{1}\right)=\left\{\mathrm{p}_{1}\right\}, \mathrm{O}\left(\mathrm{t}_{2}\right)=\left\{\mathrm{p}_{3}\right\}$, $\mathrm{O}\left(\mathrm{t}_{3}\right)=\left\{\mathrm{p}_{2}\right\} ;$ Initial Marking $M_{o}=\left[\begin{array}{lll}1 & 1 & 0\end{array}\right]$

Table 1 Basic elements of Petri Nets

\begin{tabular}{|l|c|}
\hline Name & Symbol \\
\hline Place & \\
\hline Arcs & \\
\hline Transition & \\
\hline Token & \\
\hline Marked place \\
Transition with \\
input and output
\end{tabular}

Timed Petri Nets

One major extension over the past in PN modeling is inclusion of time into the net. Time is included in a PN model; such a net is referred as Timed Petri Nets (TPNs). Time is associated with the transitions and when enabled or fired, the firing delay of the transitions can be either deterministic or stochastic in nature.

\section{i) Deterministic Timed Petri Nets (DTPNs)}

When time delays for operations or activities are fixed

\section{ii) Stochastic Petri Nets}

In this case, the process will follow some probabilistic distribution. There will be mean and spread.The timings associated with transactions are probabilistic, like Exponential, Poisson, Erlang, weibull etc. (Jerry Banks, 2005).The main stochastic distribution functions are explained below.

\section{Exponential Distribution:}

The Probability density function (pdf) for exponential distribution is $f(x)=\frac{1}{\beta} e^{-x / \beta}$ 
It is to show how stochastic times are obtained. The cumulative density function (cdf)

$\mathrm{F}(\mathrm{x})=\int_{-\infty}^{+\infty} \mathrm{f}(\mathrm{x}) \mathrm{dx}=1-\exp (-\lambda x) \quad$ where $\lambda=1 / \beta$,

Thus $\mathrm{x}=-\frac{1}{\lambda} \ln (1-\mathrm{F}(\mathrm{x})) . \mathrm{F}(\mathrm{x})=\mathrm{U}=\left[\begin{array}{ll}0 & 1\end{array}\right]$,

$\mathrm{x}=-\frac{1}{\lambda} \ln (1-\mathrm{U})$. The $\mathrm{U}$ is random which can hold any number of values in between 0 and 1 . Thus for every different value of $U$ a different value of variable $\mathrm{x}$ can be obtained. Figure 2 shows the profile of probability for fitting data. The mean time is $\beta$ and variance is $\beta^{2}$ (Law A.M, 1992)

\section{The triangular distribution ( in brief $\operatorname{Triangular}(a, b, c)$ )}

It will have probability distribution for $\mathrm{a}<=\mathrm{x}<=\mathrm{b}$.

$$
F(x)=\frac{(x-a)^{2}}{(b-a)(c-a)}
$$

And

Similarly for $\mathrm{b}<\mathrm{x}<=\mathrm{c}$

$$
F(x)=1 \frac{(c-x)^{2}}{(c-a)(c-b)}
$$

and for $\mathrm{x}>\mathrm{c} F(\mathrm{x})=1.0$; the mean is given by $\frac{(a+b+c)}{2}$

And the variance is equal to $\frac{a^{2}+b^{2}+c^{2}-a b-a c-b c}{18}$. It is the variance which is responsible for variation in task times.

There are distributions like normal distribution which can be characterized by $\mu$ and $\sigma$. The mean is $\mu$ and variance is given by $\sigma^{2}$. The cumulative density function CDF is symmetric about mean. There are many other distributions which can be used after validating the data using statistical fitting. There are promising soft wares like crystal ball, input analyser of arena to analyse the data for best fit distribution which can be used to model the problem.

\section{General Modelling Blocks in Petri Net:}

There are some fundamental building blocks used in petri nets simulation. Major ones are as follows: Sequential: One operation follows the other places and transitions are cascading as shown below in figure

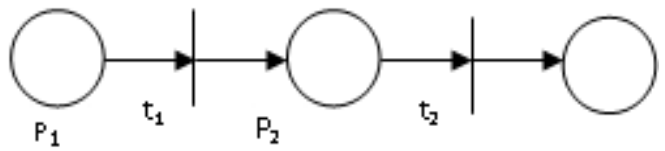

Fig.3Sequential PN

Concurrent: Two operations or more are initiated by an event. Multiple places/transitions are enabled simultaneously

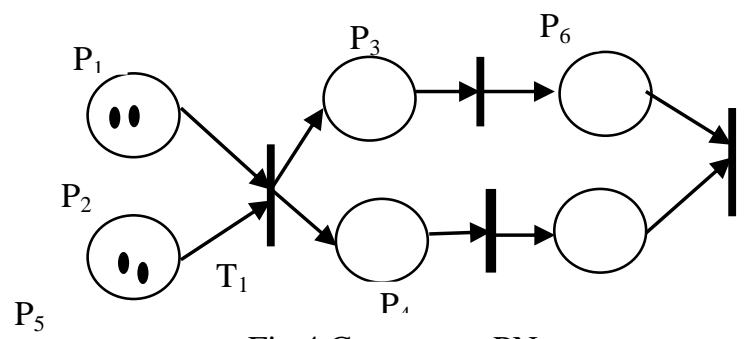

Fig.4 Concurrent PN

Mutually exclusive: Two processes can not be performed at the same time. 


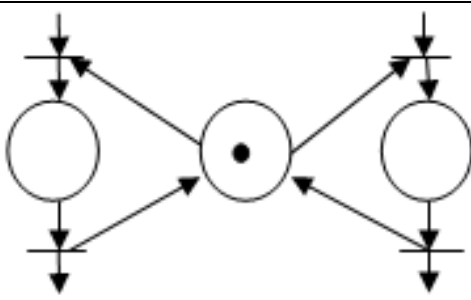

Fig.5 Mutually Exclusive PN

Buffer model: It is clear that if there is abundant raw material $\mathrm{Rm}$ available and place having $\mathrm{k}$ token are ready to fire transition $\mathrm{t}_{\mathrm{a}}$, the place $\mathrm{p}_{\mathrm{bu}}$ can at most will get $\mathrm{k}$ units of products and then place with $\mathrm{k}$ token will be empty and $t_{a}$ will stop firing

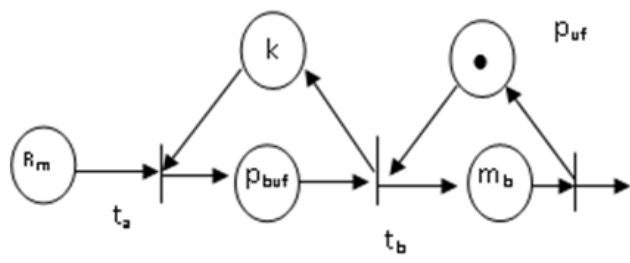

Fig.6 Buffer PN

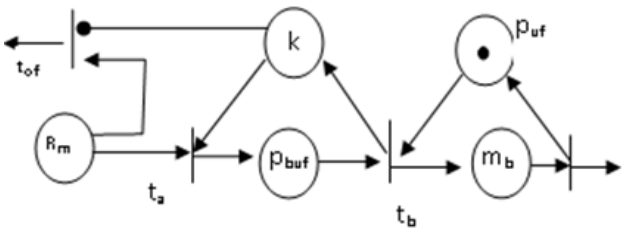

Fig.7 Overflow PN

\section{Assembly Problem.}

A product with 4 parts have been taken to explore possibilities and complexities involved in getting the optimal solutions under given conditions. The part has 4 components A, B, C, and D. It is clear from the product drawing that $\mathrm{A}$ and $\mathrm{D}$ can not be assembled in the first place. This is technical constraints. Thus the exploratory view of fist level assembly is as follows. The connectivity matrix that corresponds to the connection diagram of Figure 8 is presented in Figure 9.The maximum number of assembling options $\mathrm{K}$ in a product with $\mathrm{N}$ components at level 1 isgiven by the following expression.

$\mathrm{K}=1 / 2 \mathrm{~N} *(\mathrm{~N}-1)$. For 4 products there are $4 * 3 / 2=6$ possible options, i.e. $\mathrm{AB} ; \mathrm{AC} ; \mathrm{AD} ; \mathrm{BC} ; \mathrm{BD} ; \mathrm{CD}$. $\mathrm{AD}$ can not assembled due to technical constraints, as A and D have no possibility to be connected together.

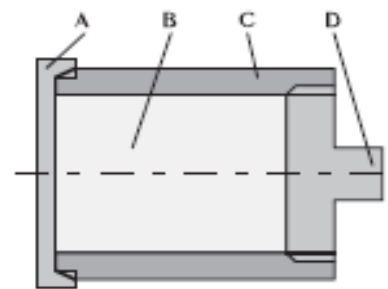

Fig.8 Product and Bill of Material

Moving to the next level, $\mathrm{ABD}$ is not possible because $\mathrm{C}$ can not be attached after wards, similarly ACD is not possible, B can not be fitted after ACD assembly. The only feasible sets are given below.

Moving to the next level, ABD is not possible because $C$ can not be attached after wards, similarly ACD is not possible, B can not be fitted after ACD assembly. The only feasible sets are given below. 


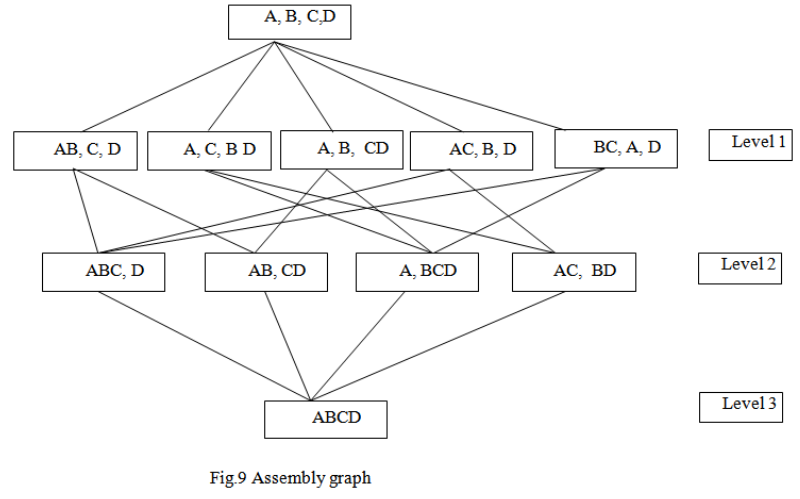

The time to assemble at first level is given below in table 2.

Table 2: The stochastic times to create assembly of any two components or subassemblies

\begin{tabular}{|l|l|l|l|l|}
\hline Task & A & B & C & D \\
\hline A & - & $\begin{array}{l}\text { AB assembly time is } \\
\text { Triangular(a, b, c) } \\
(4,5,7) \text { minutes }\end{array}$ & $\begin{array}{l}\text { AC assembly time is } \\
\text { Normal Normal }(\mu, \sigma) \\
(4,2) \text { minutes }\end{array}$ & Not feasible \\
& & - & $\begin{array}{l}\text { BC assembly time is } \\
\text { Normal }(\mu, \sigma)\end{array}$ & $\begin{array}{l}\text { BD assembly time is } \\
\text { Exponential }(\beta)=5\end{array}$ \\
\hline B & & $(4,2)$ minutes & \\
\hline C & & & - & $\begin{array}{l}\text { CD assembly time is } \\
\text { Normal distribution } \\
\end{array}$ \\
& & & $(\mu, \sigma)=(5,1)$ minutes \\
\hline
\end{tabular}

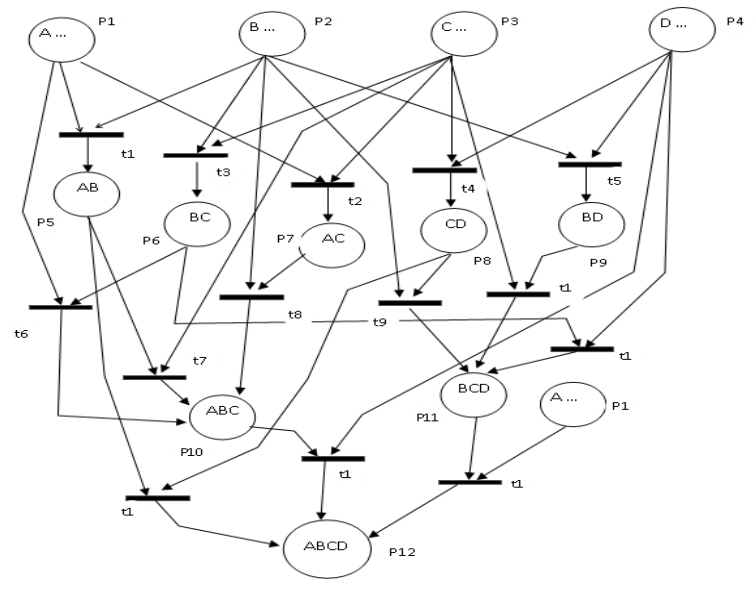

Figure 10: Assembly of Part

As we will move to the next level, $\mathrm{ABC}$ is obtained from 3 ways. i) $\mathrm{AB}$ combined with $\mathrm{C}$ ii) $\mathrm{AC}$ assembled with $\mathrm{B}$, and iii) $\mathrm{BC}$ combined with $\mathrm{A}$. These are three different alternatives may not have the same timings as set ups will be different (Thomopoulo, 1967)

\section{Simulation Of Manufacturing System}

There are many other stochastic distributions that can also be used like normal, Erlang, exponential, Poisson etc. (Averill, 1999, Geoffrey Gordon, 1992). The times must first be validated for their best fit.. A typical case of assembly has been considered for discussion and to explain as to how the Petri Net really works.

\section{Petri Net For Part Assembly:}

The generic components are Base plate (A), central part (B), side frames (C), Top cover (D).

The graphical representation for the assembly sequence is presented in figure 9 . Thus, all possible ways of doing the assembly job can be explored. Undoubtedly, the set of sequence with high throughput rate is selected for operations. The transitions will fire if input places are available with tokens. The transition will delay the time for stochastic time period and resource will remain seized during operation. The inputs places for various transitions of the petri nets are given below: 
Table 3: The input matrix I: (PxT)

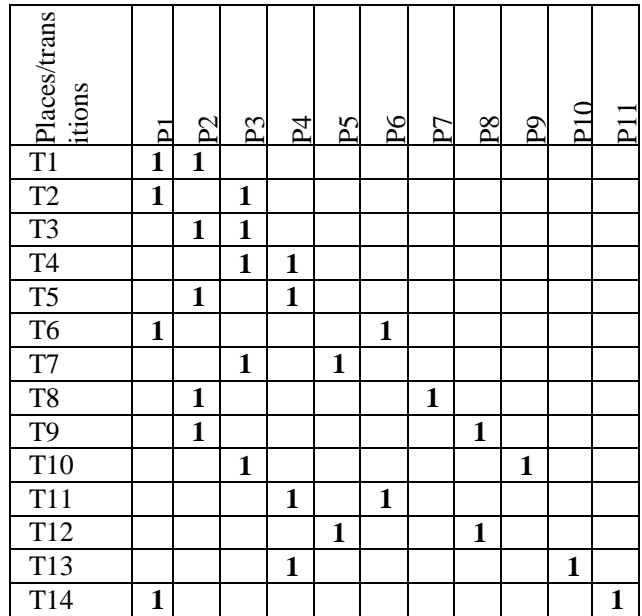

Table 5:The output matrix O: (TxP)

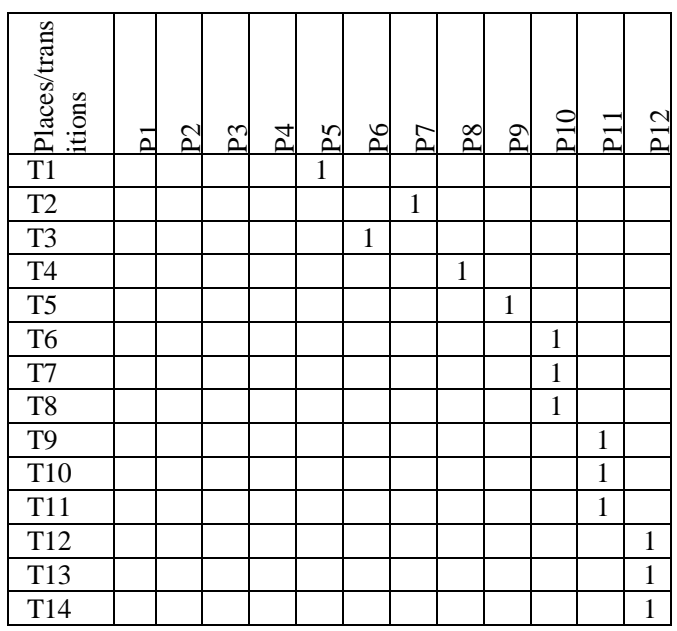

The timings for assembling $\mathrm{C}$ with $\mathrm{AB}$ is normal (6,2 minutes) and assembling $\mathrm{D}$ as last is taken as exponential (4 minute). Time for AC assembly is considered normal $(4,2)$ and assembling B with it is triangular $(5,7,9$ minutes) followed by $\mathrm{D}$. The inter arrival time for the first part to embark the assembly is considered 3.4 minutes.

The initial marking of places $m_{0}=\{n, n, n, n, 0,0,0,0,0,0,0,0\}$. Some sequences are drawn below.

Sequence \#1

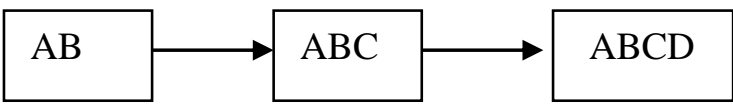

The make span $=16.35$ minutes and utilization of stations is 100,96 , and $73 \%$. Average utilizations of resources is $89.66 \%$.

\begin{tabular}{|c|c|c|c|c|c|}
\hline & Line capacity & Make span time & Resource 1 & Resource 2 & Resource 3 \\
\hline \multicolumn{2}{|c|}{ Utilization of resources } & & $100 \%$ & $96 \%$ & $73 \%$ \\
\hline Queue (time) & & & & 11.15 minutes & 1.61 minutes \\
\hline Queue (nos) & & & & 1.96 & 0.26 \\
\hline Through put & $\begin{array}{l}9.6 \text { products } / \mathrm{hr} \\
32 \text { products in } \\
200 \text { minutes }\end{array}$ & 16.35 minutes & & & \\
\hline
\end{tabular}

Sequence \#2

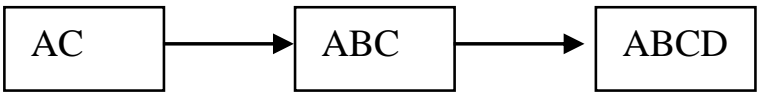

\begin{tabular}{|c|c|c|c|c|c|}
\hline & Line capacity & Make span time & Resource 1 & Resource 2 & Resource 3 \\
\hline \multicolumn{2}{|c|}{ Utilization of resources } & & $98 \%$ & $99 \%$ & $60 \%$ \\
\hline Queue (time) & & & & 0.95 minutes & 18.51 minutes \\
\hline Queue (nos) & & & & 0.13 & 5.64 \\
\hline Through put & $\begin{array}{l}8.1 \text { products } / \mathrm{hr} \\
27 \text { products in } \\
200 \text { minutes }\end{array}$ & 15.75 minutes & & & \\
\hline
\end{tabular}


Sequence \#3

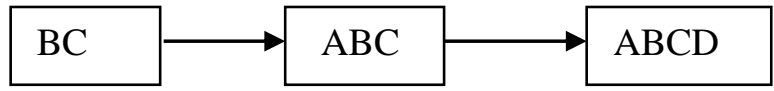

\begin{tabular}{|c|c|c|c|c|c|}
\hline & Line capacity & Make span time & Resource 1 & Resource 2 & Resource 3 \\
\hline \multicolumn{2}{|c|}{ Utilization of resources } & & $98 \%$ & $99 \%$ & $65 \%$ \\
\hline Queue (time) & & & & 22.8 minutes & 1.43 minutes \\
\hline Queue (nos) & & & & 5.28 & 0.39 \\
\hline Through put & $\begin{array}{l}8.7 \text { products } / \mathrm{hr} \\
29 \text { products in } \\
200 \text { minutes }\end{array}$ & 15.27 minutes & & & \\
\hline
\end{tabular}

The marking will start changing as the transitions will be enabled and fired. Finally, after a period P12will have accumulated a number of tokens. The sequence with maximum utilization can be chosen for implementation. The sequence \#1 is most promising for assembly operations.

\section{Simulation Results:}

The resource utilization, and make span, for sequence \#1, 2, and 3 are summarized above. In assembly line, the status of accumulation of inventory in between workstations is essential to design storage capacity or the preceding station must be forced to stop. The third sequence i.e. A-C-B-D is having long queue at station 2 and three and poor throughput. The utilization of individual station come under scrutiny as to how the line is synchronized in most stream lined manner so that the resources are utilized utmost. The work utilization is more synchronized in case of first sequence i.e. A-B-C-D. The throughput is highest 9.6 products per hour. The Sequence 1 is having station one and two almost synchronized and $100 \%$ utilized. The queuing between second and third station will not build because the workstation three is starved most of the time and thus having low utilization.

\section{Conclusions:}

The simulation results are precursor for system design. The simulation of system using Petri Net is quite convenient tool to validate different instances, scenarios, and see the performance of individual resource, queuing build up etc.. The methodology can be used for simulating any manufacturing system to yield key data like WIP, queuing, throughput, make span, utilization, output rate, etc. Petri Net can consider most realistic input to arrive at pragmatic results.

\section{Bibliography}

[1] HolzmannG.J., Design and Validation of Computer Protocols. Prentice-Hall International Editions, 1991.

[2] K.P. Valavanis, Modular Petri Net based Modeling, Analysis, Synthesis and Performance Evaluation of Production Systems, Journal of Intelligent Manufacturing, Vol. 16, pp. 67-92, 2005.

[3] Chandra and AbebayehuSeifu, Simulation and performance Analysis of manufacturing system using Deterministic and stochastic PETRI NETS, Proceedings of 3rd International conference CEETE ,Jimma University, 18-19th April 2008,

[4] Averill M. Law and Michael G. McComas, Simulation of Manufacturing Systems, Proceedings of the 1999 Winter Simulation Conference, pp.86-89, Averill M. Law \& Associates, Inc., USA, 2000.

[5] Geoffrey Gordon, System Simulation, 2nd Edition, Prentice - Hall of India, New Delhi, 1992

[6] Michel Diaz, Petri Net, John Wiley publisher, 2005.

[7] Peter Haas, Stochastic Petri Nets: Modelling Simulation, Springer Series, 2010.

[8] Law A.M. and W.D Kelton, Simulation modeling and Analysis, New York, MacGraw Hill, 1991.

[9] Jerry Banks, Discrete event Simulation, Prentice Hall, NJ, 2005.

[10] Askin, R.G. \&Standridge, C.R. (1993). Modelling and analysis of manufacturing systems; John Wiley and Sons Inc, ISBN 0-47151418-7

[11] Raouf, A., El-Sayed, E.A. and Tsui, C.L., "A New Heuristic Approach to Assembly Line Balancing", Computer and Industrial Engineering, Vol. 4, 1980.

[12] Agarwal, P.K., "The Related Activity Concept in Assembly Line Balancing", International Journal of Production Research, Vol. 23 No. 2, 1985, pp. 403-21.

[13] Bhattacharjee, T.K. and Sahu, S., "A Critique of Some Current Assembly Line Balancing Techniques", International Journal of Operations \& Production Management, Vol. 7 No. 6, 1987.

[14] Buxey, G.M., "Assembly Line Balancing with Multiple Stations", Management Science, Vol. 20 No. 6, 1974

[15] Thomopoulos, N.T., "Line Balancing — Sequencing for Mixed Model Assembly", Management Science, Vol. 14, 1967.

[16] Bhattacharjee, T.K and Sahu, A Heuristic Approach to general Assembly Line balancing, International Journal of operations and production management, 1987, Emerald Back files, 2007

[17] Seyed-Alagheb and, S. A., Ghomi, S.M. T.F., \&Zandieh, M. A simulated annealing algorithm for balancing the assembly line type II problem with sequence-dependent setup times between tasks. International Journal of Production Research,2011, 49(3), 805825.

[18] Vrat P. and Virani A., "A Cost Model for Optimal Mix of Balanced Stochastic Assembly Line and Modular Assembly System for a Customer Oriented Production System", International Journal of Production Research, Vol. 14 No. 4, 1976, pp. 445-463. 